\title{
Comparative Study of Gearbox Fault Diagnosis by Vibration Measurements
}

\author{
Dennis Hartono, ${ }^{1, a}$, Dunant Halim ${ }^{1}$, Achmad Widodo ${ }^{2}$, and Gethin Wyn Roberts ${ }^{3}$ \\ ${ }^{1}$ The University of Nottingham Ningbo China, Mechanical, Materials and Manufacturing Engineering Department, 315100 Ningbo, China \\ ${ }^{2}$ Universitas Diponegoro, Mechanical Engineering Department, 50275 Semarang, Indonesia \\ ${ }^{3}$ The University of Nottingham Ningbo China, Civil Engineering Department, 315100 Ningbo, China
}

\begin{abstract}
Vibration analysis has been demonstrated to be one of the best tools to detect faults in a gearbox by providing abundant information about the operating condition of a gearbox. However, a gearbox generates complex vibration signals, which makes it difficult to diagnose when a fault occurs. There are several fault diagnosis methods that can be utilized to analyze the underlying signals. The time-frequency method has been used and showed some promising results. On the other hand, it also has its drawback when it is applied to a complex mechanical system such as gearboxes. This paper thus attempts to examine the effectiveness of several diagnosis methods to detect faults in a gearbox from vibration measurements. The results show that the cepstrum method can provide a more accurate indication of a faulty gearbox compared to other diagnosis methods.
\end{abstract}

\section{Introduction}

The gearbox as one of the main parts of rotating machinery has been used in many engineering applications, requiring speed and torque conversions. The failure of the gear mechanism affects the entire operation of the machinery, which can cause significant loss in industry. Vibration measurement is considered as the most common method to detect failure in gears. A number of diagnosis methods have been proposed to analyze vibration signals from a defective gearbox. The frequency domain approach such as the Fast Fourier Transform (FFT) can offer a different perspective to diagnose faults in the gearbox. Theoretically it works well for stationary-signals, but not for non-stationary (transient) signals. On the other hand, the time-frequency domain, e.g Continuous Wavelet Transform (CWT), can provide a reliable diagnosis for gearboxes operating under a non-stationary condition (the run-up, run-down and transient processes) [1,2]. The simultaneous representation in time and frequency domains in a single chart can also help to provide a more detailed diagnosis of occurring faults. Unfortunately none of the methods in the time-frequency domain can provide a complete solution to indicate faults and sometimes the results can also be misleading [3].

When the faulty gear is in contact with a normal gear, a transient impulse vibration will be generated. It is well known that when the gear is faulty, sidebands can be observed around its gearmesh frequency and its

\footnotetext{
${ }^{\mathrm{a}}$ Corresponding author: dennis.hartono@nottingham.edu.cn
}

harmonics. The spacing of the sidebands indicates the location of the faulty gear [4]. However it is not an easy task to visualize such a sideband by using a conventional frequency domain method such as the Fourier Transform. This work thus compares several methods to diagnose a faulty gearbox and suggests the most effective way to diagnose the fault.

\section{Fundamental Theory}

\subsubsection{The Continuous Wavelet Transform}

The CWT is able to overcome the limitation from the FFT which only can offer global feature of the underlying signal $\mathrm{x}(\mathrm{t})$. The CWT moves data from a space to a scale domain. One of the main advantage of using CWT is its ability to provide high frequency resolution low frequency region and high time resolution in high frequency region which is very useful for gearbox fault diagnosis. The CWT of a signal $\mathrm{x}(\mathrm{t})$ is defined as a convolution integral of $\mathrm{x}(\mathrm{t})$ with dilated and scaled versions of a mother wavelet function $\Psi(\mathrm{t})[5]$ :

$$
\operatorname{CWTx}(a, b)=1 / \sqrt{a} \int_{-\infty}^{\infty} x(t) \Psi^{*}\left(\frac{t-b}{a}\right) d \tau
$$

Where $\mathrm{a}$ and $\mathrm{b}$ is the scale parameter and translation parameter respectively. Equation 1 indicates that CWT is similar to the Fourier Transform except that the CWT 
uses the family of wavelets function instead of using sine and cosine function as the basis functions.

\subsubsection{The Cepstrum}

The Cepstrum was initially used to detect echo delay times in seismic signals [6]. As mentioned in the Introduction, localized fault in the gearbox will give rise to sidebands in the vicinity of the gearmesh frequency and its harmonics. Therefore sidebands spacing contains useful information since it is related to the faulty source. The Cepstrum possess the ability to detect sidebands without a thorough inspection in frequency domain. Randal mentioned that there are 3 main applications of cepstrum analysis in gear diagnostics [4], however author believe that in Condition Monitoring of a gearbox, the main application of the Cepstrum analysis is to detect the presence of such sidebands family and its harmonics [4, 7]. Initially The cepstrum was defined as the power spectrum of the logarithm of the power spectrum. However in the area of gearbox fault diagnosis, the Cepstrum is commonly defined as the inverse Fourier Transform of the logarithmic power spectrum as defined as equation two below. Randall [4] mentioned that it is partly because it is more logical to use the inverse transform between a function of frequency and a function of time, and partly because it is then reversible to the power spectrum.

$C(\tau)=F^{-1}\{\log [\operatorname{Sxx}(f)]\}$

Where $\operatorname{Sxx}(f)$ is the power spectrum. The indepencent variable $\tau$ known as quefrency has the dimension of time(s).

\section{Comparisons of Several Fault Diagnosis Methods}

A single-stage spur gearbox was analyzed in this work. Three Accelerometers connected to the DAQ SpectraPed system were used to capture the vibration signal from the gearbox with the $5 \mathrm{kHz}$ sampling frequency. The accelerometers were mounted on one of bearings. The fault investigated in this experiment was the $50 \%$ tooth removal gear, located at the output of the gearbox with the rotational frequency of $16.67 \mathrm{~Hz}$. The goal of this experiment was to detect the particular output rotational frequency or its harmonics.

The vibration signals (in terms of the voltage output from accelerometers) from the healthy gearbox and faulty gearbox are presented in Figure 1 and Figure 2, respectively. There was a slight increase in vibration magnitude when a fault existed in the gearbox. An impulse-like response (spike) could occur when the faulty gear tooth make a contact with the other gear. However, it was difficult to identify the periodicity of this particular spike (which could correspond to the faulty gear tooth) by a direct visual inspection of the plot. It was not possible to obtain any information about location of the faulty gear tooth from the time-domain representation.

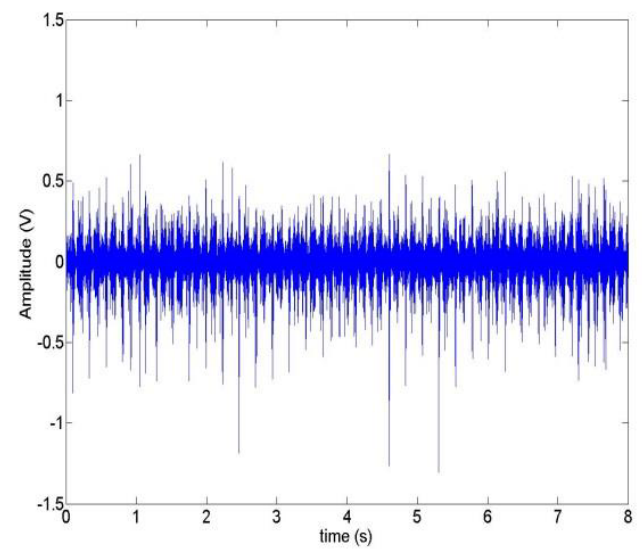

Figure 1. Vibration signal from a normal gearbox

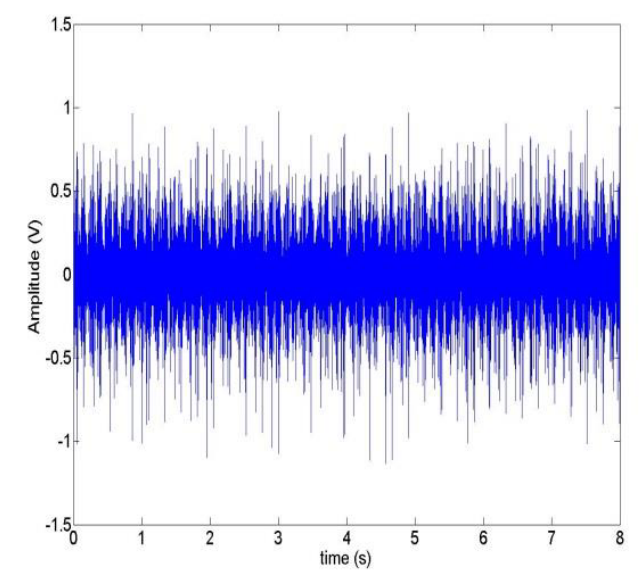

Figure 2. Vibration signal from a faultyl gearbox

The Fourier Transform offers a different representation of signals in frequency domain. It gives an indication of the faulty gearbox from the sideband spacing around the gearmesh frequency and its harmonics. The gearmesh frequency of the gearbox was $250 \mathrm{~Hz}$. However it was difficult to identify the sideband spacing from the FFT due to the signal noise as shown in Figure 3. Moreover, some sidebands could be covered by the noise, which had higher amplitudes compared to those of the sidebands. There were too many spurious peaks that also appeared in the plot that made it even harder to analyse. The spectral leakage had also an inherent drawback that exacerbated the FFT plot. Although this could be mitigated by applying a windowing function, it could not be eliminated completely. Furthermore, it was not possible to know when such a frequency existed from The Fourier Transform. It is an inherent drawback from the FFT because by default FFT utilizes sine and cosine as a basis function which means it only can provide global feature of the underlying signal which means we will lose the local feature that is represented in timedomain. To obtain such information, another method that generates the time-frequency information will be needed. 


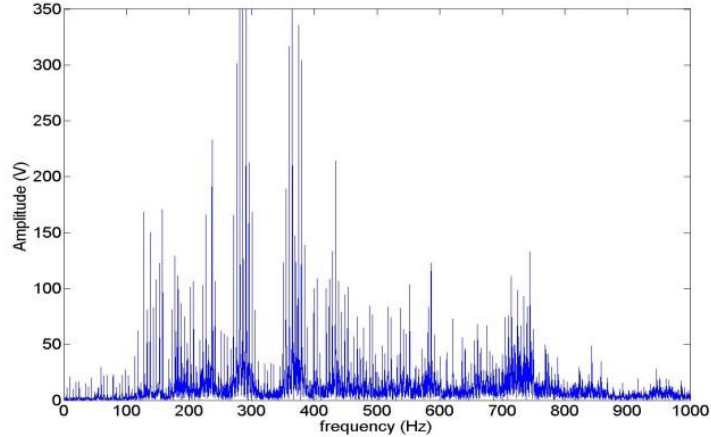

Figure 3. FFT of vibration signal for a faulty gearbox

To address the drawback of The Fourier Transform, The Continuous Wavelet Transform was investigated, for providing a time-scale (time-frequency) representation. Figure 4 shows the CWT of the normal gearbox. There was not any obvious periodicity from the figure. However it can be observed clearly that there were new generated contours for a faulty gearbox in Figure 5. The periodicity was equal to $0.24 \mathrm{~s}$. This corresponded exactly to the fourth harmonic of the faulty gearbox rotation period, which was $4 \times(60 / 1000)$ s. This periodicity can be observed more easily than the sideband spacing shown in Figure 3. The CWT scales from 11 to 21 were related to a frequency range from $193.45 \mathrm{~Hz}$ to $369.31 \mathrm{~Hz}$. From the CWT plot in Figure 5, a conclusion could be drawn that there were sidebands that appeared around the first gearmesh frequency. Although Figure 3 could also provide the same information (regarding the sideband spacing around the gearmesh Frequency), it was more difficult to identify due to the existence of noise that can mask the sidebands.

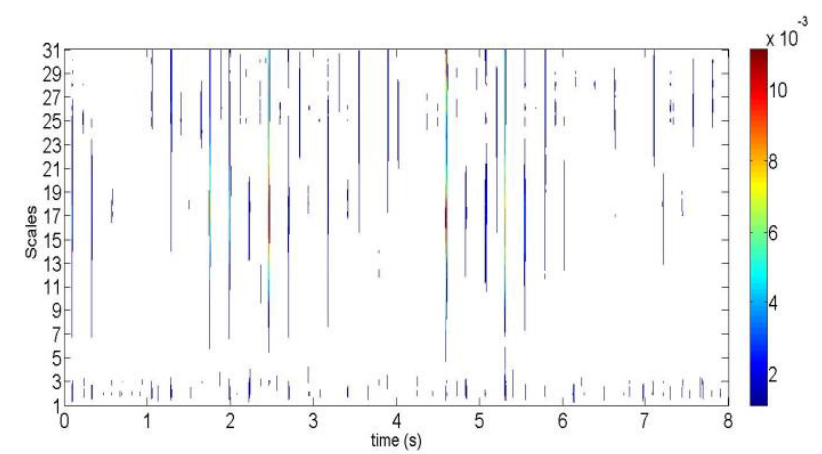

Figure 4. CWT of vibration signal for a a normal gearbox

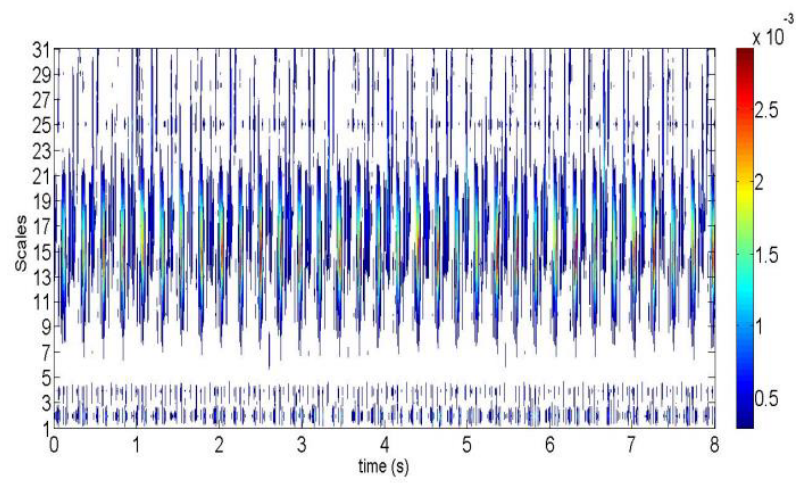

Figure 5. CWT of vibration signal for a a faulty gearbox
Finally, the cepstrum method measures the periodicity in the frequency domain (Fourier Transform)e.g. the sideband spacing around the gearmesh frequency and its harmonics[4]. The result from CWT was also supported by the cepstrum results in Fig. 6. It was obvious that there was a peak at $0.2375 \mathrm{~s}$, which corresponded exactly to the fourth harmonic of the faulty gear. Moreover, its harmonics could be observed at $0.4756 \mathrm{~s}, 0.7131 \mathrm{~s}$, and $0.951 \mathrm{~s}$. The cepstrum method provided the most direct way in detecting a fault, compared to need of a visual inspection in the CWT method.

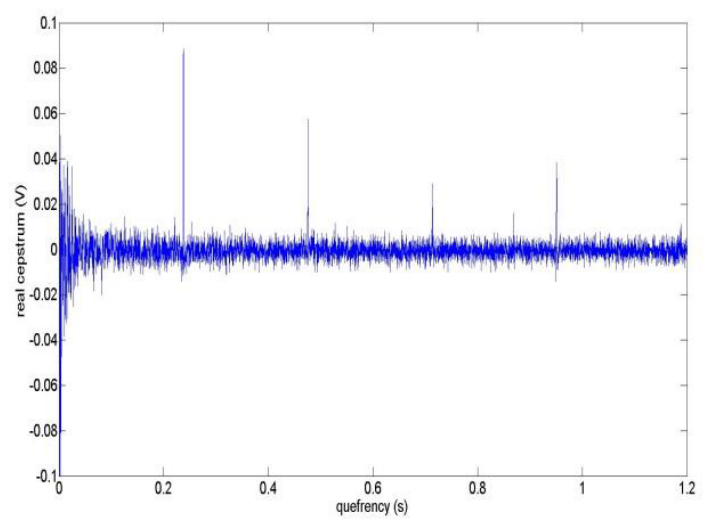

Figure 6. The cepstrum of vibration signal for a faulty gearbox

\section{Summary}

In this work, several diagnosis methods have been investigated to detect faults in a gearbox. The Fourier Transform was not informative enough to identify the faults in a gearbox, particularly due to the signal noise that masked the sidebands. The Continuous Wavelet transform, on the other hand, could provide a different perspective in time-frequency diagram. The pattern of the faulty gearbox could be directly observed from the contour of the diagram, indicating the corresponding harmonics of the faulty gear. Nevertheless, it required as visual inspection and the need to zoom in to the diagram to identify the fault indicator. The cepstrum method, however, could provide direct fault identification without the need of a thorough visual inspection of the CWT method.

\section{Acknowledgement}

The authors gratefully acknowledge the financial support provided by Ningbo Science and Technology BureauInternational Cooperation Programme (Project: 2012D10029), China.

\section{References}

1. H. Zheng, Z. Li, X. Chen, Mec Sys and Sig Proc 16, 447-457 (2002)

2. R. Yan, R. X. Gao, X. Chen, Mech Sys and Sig Proc 96(a), 1-15 (2014)

3. Feng, Z, M. Liang, Chu. F, Mech Sys and Sig Proc 38(1) 165-205 (2013) 
4. R. B. Randall, Vibration Based Condition Monitoring: Industrial, Aerospace and Automotive Applications (2011)

5. N. Baydar and A. Ball, Mech Sys and Sig Proc 17(4), 787-804 (2003)

6. B. P. Bogert, M. J. R Healy, J. W. Tukey. In Proocedings of the Symposium on Time Series Analysis, 209-243 (1963)

7. G. Dalpiaz, A. Rivola and R. Rubini, Mech Sys and Sig Proc 14(3), 387-412 (2000) 